\title{
Outcomes of Quality of Life Regarding the Next-Generation Thoracoscopic Intrapleural Hyperthermic Chemotherapy of Non-Small Cell Lung Cancer with Dissemination
}

\author{
Takanori Ayabe*, Masaki Tomita, Eiichi Chosa, Kosuke Mori, Kunihide Nakamura \\ Division of Thoracic and Breast Surgery, Department of Surgery, Faculty of Medicine, University of Miyazaki, \\ Miyazaki, Japan \\ Email: "tayabe@med.miyazaki-u.ac.jp
}

Received 3 January 2016; accepted 25 January 2016; published 28 January 2016

Copyright $@ 2016$ by authors and Scientific Research Publishing Inc.

This work is licensed under the Creative Commons Attribution International License (CC BY). http://creativecommons.org/licenses/by/4.0/

(c) (i) Open Access

\section{Abstract}

Background: We have developed a new next-generation intrapleural hyperthermic chemotherapy (IPHC) for non-small cell lung cancer with dissemination, which is a hybrid chemotherapy combined with oral S-1 medication plus conventional cisplatin-based IPHC. We now report the preliminary feasibility and outcome of quality of life (QOL) regarding this hybrid IPHC. Methods: The patient was a 76-year-old male with a $2-\mathrm{cm}$ nodule in the left upper lobe. After partial resection by video-assisted thoracic surgery (VATS), which was diagnosed with advanced pulmonary adenocarcinoma with intrapleural dissemination. We initially performed two regimens of systemic chemotherapy, S-1 (day $1-21,100 \mathrm{mg} \mathrm{2X/day)} \mathrm{+} \mathrm{CDDP} \mathrm{(day} \mathrm{8,} 60 \mathrm{mg} / \mathrm{m}^{2}$ ) and S-1 (day $1-14,100$ mg 2X/day) + CBDCA (day 1, AUC 5). The regimen of next-generation IPHC is oral S-1 medication (day $1-21,100 \mathrm{mg} /$ day) + intrapleural hyperthermic perfusion of cisplatin $\left(200 \mathrm{mg} / \mathrm{m}^{2}\right)$ with VATS (day $8,43^{\circ} \mathrm{C}, 2$ hours). Adverse outcomes, QOL, and pleural effusion were assessed in three regimens. To investigate the outcomes of the QOL, the European Organization for Research and Treatment of Cancer QOL Questionnaire (EORTC QLQ-C30 and QLQ-LC13), the QOL questionnaire for cancer patients treated with anticancer drugs (QOL-ACD), the Cancer Dyspnea Score (CDS), and the St. George's Respiratory Questionnaire (SGRQ) were used. Results: During the IPHC treatment course, grade 3 neutropenia, anemia, and diarrhea were observed. The physical function after IPHC became worse compared to that before the IPHC. Fatigue during chemotherapy (CBDCA+S-1) was more pronounced than that during the IPHC. Nausea, vomiting, and diarrhea during the IPHC were prevalent than those of chemotherapy. The overall QOL after the IPHC was improved compared to that before the IPHC. Regarding before and after the IPHC, the physical function after the

${ }^{*}$ Corresponding author.

How to cite this paper: Ayabe, T., Tomita, M., Chosa, E., Mori, K. and Nakamura, K. (2016) Outcomes of Quality of Life Regarding the Next-Generation Thoracoscopic Intrapleural Hyperthermic Chemotherapy of Non-Small Cell Lung Cancer with Dissemination. Journal of Cancer Therapy, 7, 63-77. http://dx.doi.org/10.4236/jct.2016.71008 
IPHC became worse compared to that before the IPHC, on the other hand, the global QOL before and after the IPHC had not dramatically changed. Pleural effusion was controlled after the IPHC for more than 1 year. Conclusion: The first case of a clinical trial of the next-generation IPHC showed grade 3 adverse events. However, it was an acceptable feasibility compared to the usual platinum doublet chemotherapy. The effectiveness of the IPHC allowed the patient to obtain a good control of the pleural effusion and preserved the patient's QOL.

\title{
Keywords
}

\author{
Non-Small Cell Lung Cancer, Intrapleural, Hyperthermic, Chemotherapy, Cisplatin, S-1, Quality of \\ Life
}

\section{Introduction}

Lung cancer remains a leading global cause of cancer mortality and morbidity [1]. In Japan, the number of operations for primary lung cancer in 2013 was 37,008 [2]. Among lung cancer subtypes, adenocarcinoma comprises an overwhelming percentage of $69.1 \%$ of the total lung cancer surgery, followed by squamous cell carcinoma of $19.9 \%$. Lobectomy is performed in 27,469 patients, which is $74.2 \%$ of the entire cases. Patients with lung cancer should receive tailored treatment based on the histological subtype of the tumor because of disparities in tumor aggressiveness and the variable response to treatment. Most patients present with advanced disease, and modest gains in survival, symptom control, and quality of life attained with chemotherapy.

By the seventh edition of the tumor-node-metastasis(TNM) staging system for lung cancer [3], an advanced lung cancer with pleural dissemination and/or malignant pleural effusion, which is defined as M1a and described in "tumor with pleural nodules or malignant pleural/pericardial effusion", is displayed as stage IV, the same as advanced cases with a distant metastasis. This type of lung cancer is considered to have no indication for curative surgery, and the aggressive and invasive therapy should be difficult because of the patient's poor performance status and worse quality of life (QOL), and the therapeutic effective and appropriate treatments have not yet been established. Generally, its prognosis should be worse as a few optional treatments, i.e., cytotoxic systemic chemotherapy, and molecular-targeted drug treatment will be performed.

Based on the basic scientific study of the effect of the anti-cancer effect of intrapleural hyperthermic chemotherapy (IPHC), our study group has performed the IPHC in clinical practice scince 1980. From 1988, our IPHC has been used for lung cancers with dissemination with resectable cases of primary lung cancer with no lymphatic metastasis and no distal metastasis, which are possible for single-lung ventilation under general anesthesia. Until 2010, our conventional IPHC was performed as a clinical treatment [4]-[7].

However, there has not been a distinct clinical phase-II study and no new evidence has been obtained and updated for the IPHC. The regimen is a conventional, single use of cisplatin and its hyperthermic perfusion for the intrapleural space. New evidence regarding the efficacy of the IPHC should be obtained. We develop a new next-generation IPHC, whose regimen consists of platinum doublet chemotherapy with the new 3rd generation cytotoxic drugs that is the additional oral medication of S-1 drugs.

The recent treatment outcome of unresectable advanced lung cancer showed a three-fold extended survival (more than 2 to 3 years) longer than that of the old conventional chemotherapy, because of the newly obtained evidence of platinum doublet chemotherapy with the 3rd generation cytotoxic anticancer drugs and molecular targeted drugs. It is a very important issue regarding the management of the symptoms during palliation of the malignant pleural effusion with carcinomatosis.

As not only for a passive therapy, but also an aggressive palliation therapy for the malignant pleural effusion, the cytotoxic treatment of disseminated tumor cells will give the patient a longer progression-free treatment along with a good QOL. To extend the survival period and to improve symptoms, such as dyspnea, and also to maintain the quality of life during the long-term survival term even though carrying tumors, our new next-generation IPHC treatment will become one of the optional, aggressive, and effective treatments.

As the optimal aggressive palliation therapy, our new clinical trial involving the next-generation IPHC is a hybrid chemotherapy combined with oral S-1 medication plus conventional cisplatin-based IPHC for the advanced thoracic malignancies with pleural effusion. We report the first case, benefit, and the preliminary feasi- 
bility of safety, efficacy, and quality of life.

\section{Methods}

\subsection{Patient Profile}

The patient was a 76-year-old male with chest pains who had a 2-cm nodule in the left upper lobe (Figure 1(a)). A bronchoscopy was performed, but lung cancer could not be pathologically diagnosed. He was admitted to our hospital, after which the chest computed-tomographic (CT) scanning displayed a $22 \times 18 \mathrm{~mm}$ irregular mass with spicula, pleural indentation, air bronchogram in the left $\mathrm{S}^{1+2}$ of the upper lobe, in which the tumor was located on the surface of viscera pleura, and a small multiple nodule was found in the interlobe pleura and a localized small amount of pleural effusion was suspected (Figure 1(b), Figure 1(c)). These findings suggested dissemination and pleuroitis with malignant pleural effusion (T1bN0M0, or M1a). A positron emission tomography-CT showed an abnormal accumulation of the tumor (standardized uptake value maximum, early: 6.03, delay: 8.18) and lung cancer was suspected, however, there was no accumulation of mediastinal lymph nodes and distant metastasis. Although lung cancer was suspected (Lt., U, $\mathrm{S}^{1+2}, 22 \times 18 \mathrm{~mm}$, T1bN0M0, c-stage IA, M1asuspected), a lung biopsy was performed by video-assisted thoracic surgery (Figure 2(a)-(c)), and the intraoperative prompt pathology showed advanced pulmonary adenocarcinoma with intrapleural dissemination (Figure 2(b)). The permanent pathology of the specimen showed a mixed subtype adenocarcinoma, pleura invasion (Lt. $\mathrm{U}, \mathrm{S}^{1+2}$, Adenocarcinoma, mixed subtype, $20 \times 15 \mathrm{~mm}$, pl2, G2, ly0, v0, pT3, cNx, pM1a, p-stage IV) (Figure 2(d)). A mutation analysis of the epidermal growth factor receptor gene was negative.

\subsection{Systemic Platinum Doublet Chemotherapy}

We performed two regimens of systemic chemotherapy before the IPHC. The first cycle of chemotherapy was S-1 (tegafur/gimeracil/oteracil potassium, TS- $1^{\circledR}$, Taiho Pharmaceutial Co. Ltd., Tokyo, Japan) (day 1 - 21, 100 mg 2X/day) plus CDDP (cisplatin, Platosin ${ }^{\circledR}$, PhizerJapan Inc., Tokyo, Japan) (day 8,60 mg/m², intravenous drip infusion) in August 2014, and the second cycle of chemotherapy was S-1 (day 1 - 14, $100 \mathrm{mg}$ 2X/day) plus CBDCA (carboplatin, Paraplatin ${ }^{\circledR}$, Bristol-Myers K.K., Tokyo, Japan) (day 1, AUC 5, intravenous drip infusion) in October, 2014.

\subsection{Surgery for the New Next-Generation IPHC}

This study was approved by the University of Miyazaki Hospital Clinical Research Ethics Board (No. 2014 091, UMIN000015343) in October 2014. We gave informed consent to the patient in this study. The new next-generation IPHC is a hybrid chemotherapy, which is S-1 (day $1-21,100 \mathrm{mg} /$ day) plus IPHC with VATS (day $8,43^{\circ} \mathrm{C}$, 2 hours, intrapleural hyperthermic perfusion of cisplatin $\left(200 \mathrm{mg} / \mathrm{m}^{2}\right)$ were performed in November 2014. On day 1 (7th preoperative day), S-1 was orally administered twice a day [total dose: $80 \mathrm{mg} /$ day, body surface area (BSA) $<1.25 \mathrm{~m}^{2} ; 100 \mathrm{mg} /$ day, $1.25<\mathrm{BSA}<1.5 \mathrm{~m}^{2} ; 120 \mathrm{mg} /$ day, BSA $>1.5 \mathrm{~m}^{2}$ ]. On day 8 , we performed the IPHC $\left(43^{\circ} \mathrm{C}, 2\right.$ hours, $200 \mathrm{mg} / \mathrm{m}^{2}$ of cisplatin), the therapy of which was based on the modified

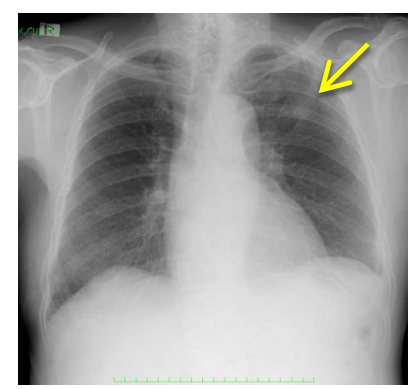

(a)

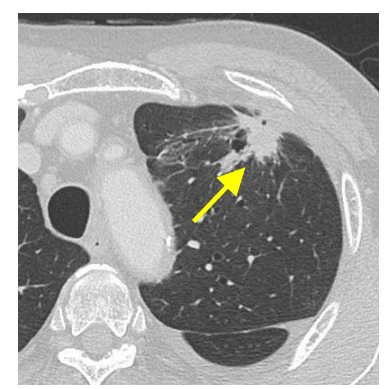

(b)

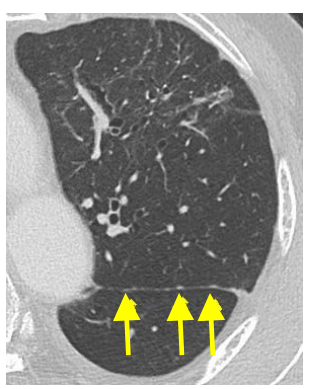

(c)

Figure 1. (a) An abnormal shadow was observed in the left upper lung field. (b) A $22 \times 18$ $\mathrm{mm}$ irregular mass located in near pleural membrane in $S^{1+2}$. (c) Small nodules were observed in interlobe pleura of upper and lower lobes with small amount of pleural effusion, which were suspected to be adissemination. 


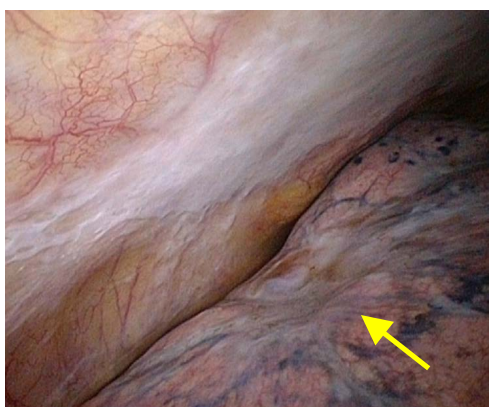

(a)

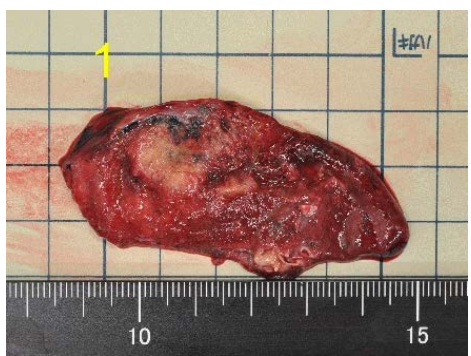

(c)

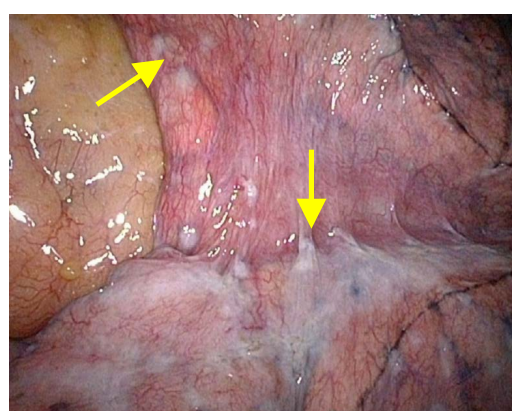

(b)

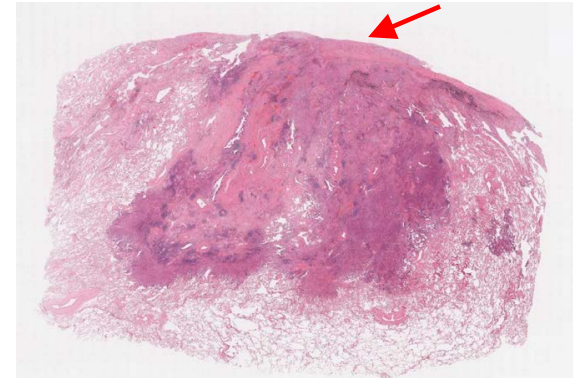

(d)

Figure 2. (a) A video-assisted thoracoscopic intraopeartive finding. A yellow arrow points out the surface of the primary tumor of the left upper lobe. (b) There are a lot of small white nodes in the interlobe of upper and lower lobes, which were diagnosed with dissemination confirmed by prompt intraoperative pathological examination. (c) After partial resection of the tumor of upper lobe, the cut surface of the primary tumor $(20 \times 15$ $\mathrm{mm}$ ). (d) A macroscopic pathological finding. The mixed subtype adenocarcinoma (lepidic growth 10\%) invaded the pleural surface, pT3, $20 \times 15 \mathrm{~mm}, \mathrm{pl} 2, \mathrm{G} 2$, ly0, v0).

methods from the previous reports of Matsuzaki et al. [4]-[6]. The new hybrid next-generation IPHC was expected to have a triple synergistic effectiveness of cisplatin and S-1 cytotoxic and hyperthermic anti-tumor effects on the tumor cells. Under general anesthesia, after a single lung ventilation, the IPHC was performed under VATS. A thoracovideoscope was inserted in the left pleural cavity. Two ports were inserted at the lateral thorax. Two inflow and outflow tubes were placed in the pleural cavity, while an intrapleural thermometer and air-vent tube were inserted in the intrathorax thorough port wound for the thoracoscopy, which were connected to a specially designed circuit (modified CRPH-3000C, MERA, Ltd.).A $150 \mathrm{mg}$ amount of fosaprepitant megluminein in $100 \mathrm{ml}$ of saline was intravenously dripped for 30 minutes. A $0.75 \mathrm{mg}$ amount of palonosetron hydrochloride and $16 \mathrm{mg}$ of examethasone in $50 \mathrm{ml}$ of saline was intravenously dripped for 10 minutes. These drugs were prophylactically used to treat nausea and vomiting. The thoracic cavity was irrigated for 2 hours with a $43^{\circ} \mathrm{C}$ saline solution $\left(3000 \mathrm{~mL}\right.$ ) containing $200 \mathrm{mg} / \mathrm{m}^{2}$ of cisplatin. At the end of the perfusion, all the fluids in the thoracic cavity were removed. The irrigated thoracic cavity was then washed with saline. When a one-lung-ventilation is converted to a two-lung one, $1 \mathrm{~g}$ of methylprednisolone sodium succinate in $100 \mathrm{ml}$ of saline was intravenously dripped at the expansion of collapsed lung to protect the reperfusion pulmonary injury.

\subsection{Measurement of Oxidative Stress and Anti-Oxidant Capacity during IPHC}

The serum reactive oxygen metabolite (ROM) level was measured as an indicator of the oxidative stress using a spectrophotometric method; given that hydroperoxides are intermediate oxidation products of lipids, peptides, and amino acids, the overall oxidative stress can be spectrophotometrically estimated by measuring the total level of hydroperoxides [8].

Blood samples were collected just before the operation, and at the IPHC base line, the 1-hour IPHC, the 2-hour IPHC, and the release of one-lung ventilation, which were immediately centrifuged at $3000 \mathrm{rpm}$ for 15 min and intraoperatively analyzed. The separated serum was immediately used for the derivatives of the ROM (D-Roms) test and the biological antioxidant power test (BAP) as described below. 


\subsubsection{The D-Roms Test}

In order to measure the quantity of serum oxidative stress, the total amount of organic hydroperoxides in the serum was spectrophotometrically measured using the D-Roms test [9] [10]. Briefly, $20 \mu 1$ of serum was dissolved in an acetate-buffered solution ( $\mathrm{pH} 4.8)$. The hydroperoxide groups (R-OOH) react with the transition metal ions $\left(\mathrm{Fe}^{2+}, \mathrm{Fe}^{3+}\right)$ liberated from the proteins in the acidic medium and are converted to alkoxy (R-O•) and peroxy radicals (ROO•), according to the Fenton reaction. These newly formed radicals, the quantities of which are directly proportional to those of the peroxides present in the serum, are chemically trapped with a chromogen $(\mathrm{N}$, $\mathrm{N}$-diethyl-para-phenylendiamine), leading to the formation of the corresponding radical cation. The concentration of this persistent species can be determined by spectrophotometric procedures (detecting its absorption at $505 \mathrm{~nm}$ ). The concentrations are expressed in conventional units (Carratelli units; UCarr) in which 1 UCarr corresponds to $0.8 \mathrm{mg} / \mathrm{H} \mathrm{H}_{2} \mathrm{O}_{2}$. The D-Roms test was performed using a FRAS 4 analyzer (Health \& Diagnostics Limited Co., Italy). The reagents for the D-Roms test were purchased from Wismerll Co., Ltd. (Tokyo, Japan).

\subsubsection{The BAP Test}

In order to assess the antioxidant capacity (AOC) in the serum, the ferric-reducing ability was examined using the BAP test [11]. This test uses a colored solution containing a source of ferric ions bound to a chromogenic substrate, which becomes decolored when the ferric ions $\left(\mathrm{FeCl}_{3}\right)$ are reduced to ferrous ions $\left(\mathrm{FeCl}_{2}\right)$ by the serum sample. The AOC is expressed in comparison to a lyophilized human control serum with known activity $(\mu \mathrm{mol} / \mathrm{l})$. Briefly, ferric chloride was mixed with a chromogen substrate, a thiocyanate derivative. Serum $(10 \mu \mathrm{l})$ was dissolved in this reaction mixture and incubated at $37^{\circ} \mathrm{C}$ for $5 \mathrm{~min}$. The reduction of the ferric ion was quantified by measuring the absorbance change at $505 \mathrm{~nm}$, and this indicated the antioxidant activity of the serum sample. The powers of the antioxidant activity were expressed by comparison to a lyophilized human control serum with a known activity ( $\mu \mathrm{mol} / \mathrm{l})$. The BAP assays were also performed by a FRAS 4 analyzer. The reagents for the BAP test were purchased from Wismerll Co., Ltd (Tokyo, Japan).

\subsection{Adverse Outcomes}

The patient was monitored weekly throughout the treatment by physical examination, recording of the toxic effects, complete blood cell counts, and blood chemistry. The patient was examined for adverse events. The adverse events were evaluated after the completion of the chemotherapy according to the Common Terminology Criteria for Adverse Events Version. 4.0 (CTCAE v4.0). The CTCAE v4.0 displays Grades 1 through 5 with unique clinical descriptions of severity for each adverse event (AE) based on the following general guideline: Grade 1, mild AE; Grade 2, moderate AE; Grade 3, severe AE; Grade 4, life-threatening or disabling AE; Grade 5 , death related to $\mathrm{AE}$.

\subsection{The Patient-Centered Outcomes of Quality of Life}

The QOL data were collected by using questionnaires from the European Organization for the Research and Treatment of Cancer Quality of Life Questionnaire (EORTC QLQ-C30/LC-13), the quality of life questionnaire for cancer patients treated with anticancer drugs (QOL-ACD), Cancer Dyspnea Scale (CDS) for assessing dyspnea, and the St. George's Respiratory Questionnaire (SGRQ) score. The EORTC QLQ-C30/LC-13 and QOL-ACD were used to compare the patient-centered QOL of chemotherapy of CDDP+S-1, CBDCA+S-1, and IPHC. The CDS and the SGRQ were used to measure the QOL regarding the respiration system during the IPHC treatment course.

\subsubsection{Health-Related Quality-of-Life (HR-QOL) Assessment}

The QOL was assessed by the EORTC QLQ-C30, a well-validated and widely used QOL tool [12] [13] and with a trial-specific symptom checklist; the lung cancer questionnaire module, QLQ-LC-13, was also used. The EORTC QLQ-C30/LC-13 is a standardized, self-administered disease-specific HR-QoL instrument designed for use in estimating the HR-QoL of oncologic patients. It contains the core questionnaire C30 and the supplementary module LC-13 specifically designed for use with lung cancer patients. The C30 is composed of 9 multi-item scales including 5 functional scales, 3 symptom scales, a global quality-of-life scale, and 6 single-item symptom measures. The LC-13 contains 1 multi-item and 9 single-item symptom scales assessing both the symptoms of the cancer and the adverse effects of the treatments. All the items are scored on a scale of 0 to 100 , with a high 
score on a functional or the global quality-of-life scale representing a high level of functioning or quality of life and a high score on a symptom scale representing a high level of symptoms.

\subsubsection{The Quality of Life Questionnaire for Cancer Patients Treated with Anticancer Drugs (QOL-ACD)}

The QOL-ACD consisting of four domains (functional, physical, mental, and psychosocial) and a global face scale, was developed as a generic questionnaire for Japanese cancer patients undergoing chemotherapy [14]. T. Matsumoto et al. [15] examined the validity and reliability of this questionnaire for Japanese patients with advanced non-small-cell lung cancer (NSCLC), who participated in two randomized phase III trials. The results confirmed the high reliability of the questionnaire. The results of a factor analysis provided strong support for the domain structure used in the questionnaire. Each of the four domains had a moderate to strong association with important clinical variables, such as performance status or weight loss, and a correlation analysis showed that the face scale provided an appropriate measure of the global quality of life. The QOL-ACD is potentially useful for the clinical research of Japanese patients with advanced NSCLC.

\subsubsection{Cancer Dyspnea Scale (CDS) for Assessing Dyspnea}

Dyspnea is one of the most frequent and refractory symptoms in cancer patients. The CDS was translated into English by the standard 'forward-backward"' translation method [16]-[18] by Tanaka et al. [19]; this translation produced the version of the CDS-E originally described and published in English in 2000. This instrument assesses breathing difficulty in the few days preceding its administration. It consists of 12 questions, scored on five-point Likert scales ranging from "not at all" to "very much". The questions are grouped into three factors; i.e., sense of effort, sense of anxiety, and sense of discomfort. The original CDS used the time period of "a few days" without further definition. To operationalize this, the subjects were instructed to consider "a few days" as "within the past seven days". The CDS is a brief, valid and feasible scale for assessing the multidimensional nature of dyspnea in cancer patients.

\subsubsection{The St George's Respiratory Questionnaire (SGRQ) Score}

The SGRQ is a standardized, self-administered questionnaire for measuring impaired health and perceived HR-QoL in airway diseases [20]. It consists of 76 items, producing a "symptoms", an "activity", an "impact" and a "total score". The "symptoms score" assesses the patients' perception of their recent (4 weeks) respiratory problems; the "activity" score measures the patients' current disturbance to perform daily physical activity; the "impact" score evaluates the whole range of disturbances the patients currently experience in their life due to respiratory problems and the "total" score sums and weighs all the former components. The scores can range from 0 (no impairment) to 100 (the worst impairment) for each component; higher scores denote greater distress and thus, a worse HR-QoL. The questionnaire was administered and scored according to the instruction manual.

\subsection{Treatment Evaluation}

The effectiveness of the control of pleural effusion was evaluated by chest CT scanning. The degree of pleural effusion was measured as the height from the back chest bottom at the same slice level.

\section{Results}

\subsection{Clinical Postoperative Course, Adverse Event, and Outcomes of the Patient}

After the first cycle of chemotherapy [S-1 (day 1 - 21,100 mg, 2X/day) plus CDDP (day 8, $60 \mathrm{mg} / \mathrm{m}^{2}$, intravenous drip infusion)], the adverse event was grade 3 anemia. After the second cycle of chemotherapy [S-1 (day 1 14, $100 \mathrm{mg}, 2 \mathrm{X} /$ day) plus CBDCA (day 1, AUC 5, intravenous drip infusion)], the adverse event was grade 3 anemia. A blood transfusion was performed (Red cell Concentrates, 2U). In November, 2014, S-1 (100 mg/day, day 1) was started by oral medication. On the day 8 , under general anesthesia, the next-generation IPHC was performed as a hybrid chemotherapy [S-1 (day 1 - 21, $100 \mathrm{mg}$ /day) plus IPHC with VATS [day8, $43^{\circ} \mathrm{C}, 2$ hours, intrapleural hyperthermic perfusion of cisplatin $\left.\left(200 \mathrm{mg} / \mathrm{m}^{2}\right)\right]$. Figure 3(a)-(c) shows the status of the pleural surfaces during the treatment course. Figure 3(a) showed a dissemination, which was identified as multiple white nodules during the initial diagnostic VATS before two regimens of systemic chemotherapy. Figure 3(b) showed the status of the changed pleural surface with a whity-thickness after two regimens of systemic chemo- 


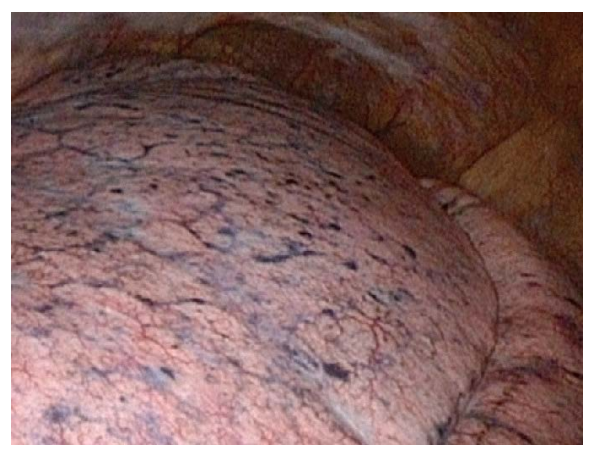

(a)

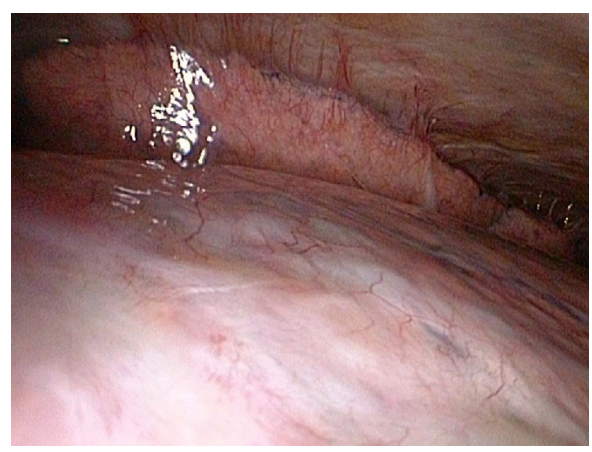

(b)

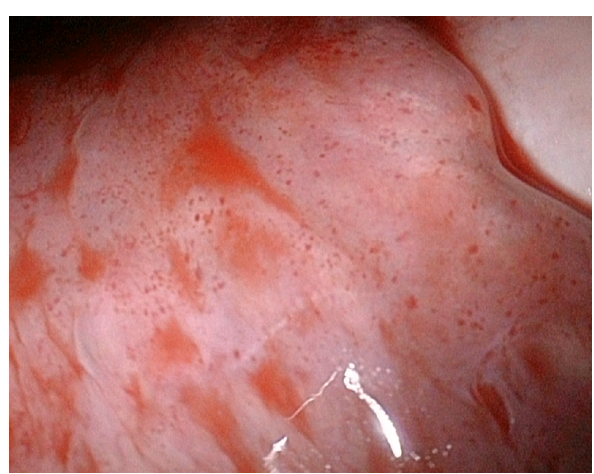

(c)

Figure 3. Transition of pleural surface changes before chemotherapy and after IPHC. (a) The first operative findings of video-assisted thoracic surgery for diagnosis, which is chemo-naïve pleural surface findings before chemotherapy (in July, 2014). (b) After 2 regimens of systemic chemotherapy, which is IPHC-naïve pleural surface findings. The pleura has been changed whitey-thickness due to two systemic chemotherapies. (c) After 2 hours of irrigation with a $43^{\circ} \mathrm{C}$ saline solution ( $3000 \mathrm{~mL}$ ) containing $200 \mathrm{mg} / \mathrm{m}^{2}$ of cisplatin. The pleural surface showed reddish, injected, edematous, and small red-spot bleeding changes.

therapy before starting the IPHC. Figure 3(c) showed the reddish, injected, and edematous pleura at the finish after 2 hours of IPHC treatment. On the 4th IPHC postoperative day (4th POD, on day 11), the chest drainage tube was removed (65 ml/day). For grade 2 nausea, ondansetron hydrochloride hydrate $(4 \mathrm{mg})$ was given and an intravenous drip infusion (1000 ml/day) was performed. On the 5th POD (on day 12) for the grade 3 digestive disturbance, the oral S-1 medication was stoppted for 3 days. Hangeshashinto (TJ-14, Japanese Kampo drug) and loperamide hydrochloride were given to the patient for the grade 3 diarrhea. On the 8th POD (on day 15), the reduced oral S-1 (80 mg/day, 80\% reduction of the initial dose) had been completed as medication to the patient until the 16th POD (on day 24); the final dose intensity of the oral S- 1 was $84.7 \%$ (1780 mg/2100 mg). On the 15th POD (on day 23), a blood transfusion (Red Cell Concentrates, 2U) was performed for the grade 3 anemia (Hb $6.7 \mathrm{~g} / \mathrm{dL}$ ). On the 18th POD (on day 26), granulocyte-colony stimulating factor (100 $\mu \mathrm{g} /$ day, for 5 days) was injected for grade 3 neutropenia. There were no further events and the patient was discharged.

\subsection{Oxidative Stress and Anti-Oxidant Capacity during IPHC}

The serum oxidative stress level during the IPHC (Figure 4) decreased according to the time course of the IPHC compared to the value before the IPHC operation. During anesthesia and 2 hours of IPHC, the base line, the 1-hour, and 2-hour values had slightly decreased. For the 1-hour value during the IPHC treatment, which had decreased the most (356 U-Carr), while the 2-hour value had not decreased, and remained constant. After finishing of the 2-hour IPHC treatment, the value after release of the one-lung ventilation increased, the value of which had rather more significantly increased compared to that of the base line value.

The serum antioxidant capacity level during the IPHC (Figure 5) also gradually decreased until 1-hour of the IPHC, which depended on the time course of the IPHC treatment. The value $(235 \mu \mathrm{mol} / 1)$ after the 2-hour IPHC 


\section{Serum oxidative stress (U-Carr)}

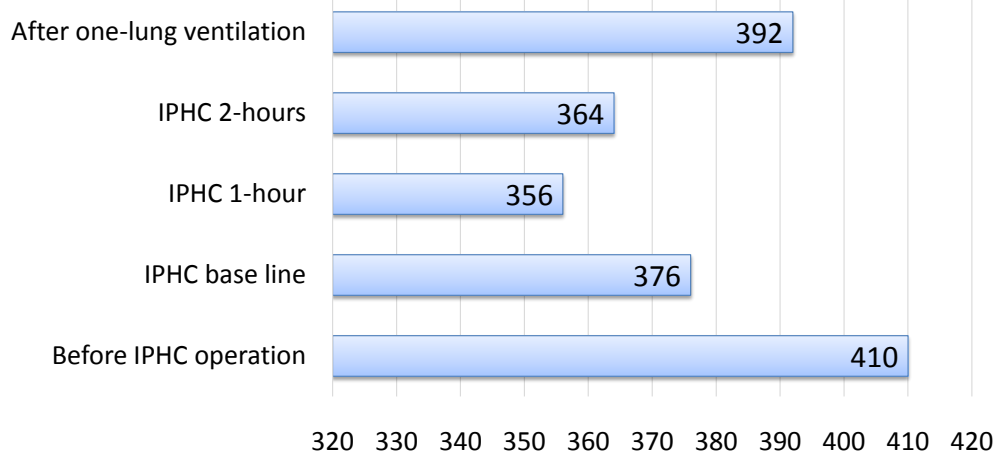

Figure 4. Serum oxidative stress (U-Carr).

Serum antioxidant capacity $(\mu \mathrm{mol} / \mathrm{l})$

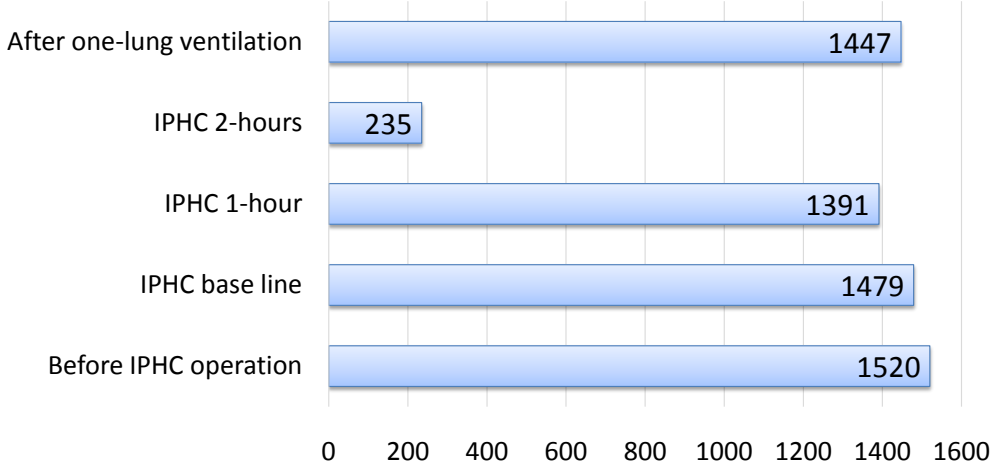

Figure 5. Serum antioxidant capacity $(\mu \mathrm{mol} / \mathrm{l})$.

decreased the most compared to the values before the IPHC base line. During the release of the one-lung ventilation, the serum antioxidant capacity increased, then recovered to the preoperative level.

Based on these results, the degree of the serum oxidative stress during the IPHC treatment had not significantly changed, but remained almost the same status. On the other hand, the degree of the serum anti-oxidant capacity had dramatically decreased at the end of the 2-hour IPHC treatment, while the anti-oxidant capacity was decreased due to the oxidative stress of anesthesia and IPHC, but recovered after finishing of the IPHC.

\subsection{Patient-Centered Outcomes of Quality of Life}

\subsubsection{Outcomes of EORTC QLQ-C30/LC-13}

Figure 6 shows the QOL scores of the functional scales and global QOL. The level of the five functional domains (physical, role, emotional, cognitive, and social) and the global QOL among the chemotherapies of $\mathrm{CDDP}+\mathrm{S}-1$ (on day 9), CDDP+S-1 (on day 12), and IPHC (CDDP+S-1) (pre-IPHC, day 7, day 21, day 42, 4month, 7-month, and 9-month) were evaluated and compared. Although the IPHC treatment had stress due to the general anesthesia and surgical physical load, by comparison with the other chemotherapy of CDDP+S-1 and CBDCA+S-1, the physical domains showed worse score values. The degrees of the QOL regarding the role, cognitive, emotional, and social domains showed similar score values. The physical and emotional domain showed worse score values than those of the role, cognitive, and social ones. However, on the global QOL, the score values showed similar degrees. Based on the outcomes from the EORTC QLQ-C30 before and after the IPHC, the physical function after the IPHC became worse compared to the status before the IPHC, on the other hand, the global QOL before and after the IPHC had not changed.

Figure 7 shows the QLQ-C30 (symptom scales and single items). The symptom of fatigue showed the worse score values compared to the other symptom scales among the three chemotherapeutic treatments. The regimen of CBDCA+S-1 chemotherapy showed the highest score value in all treatments. The symptoms of nausea, 


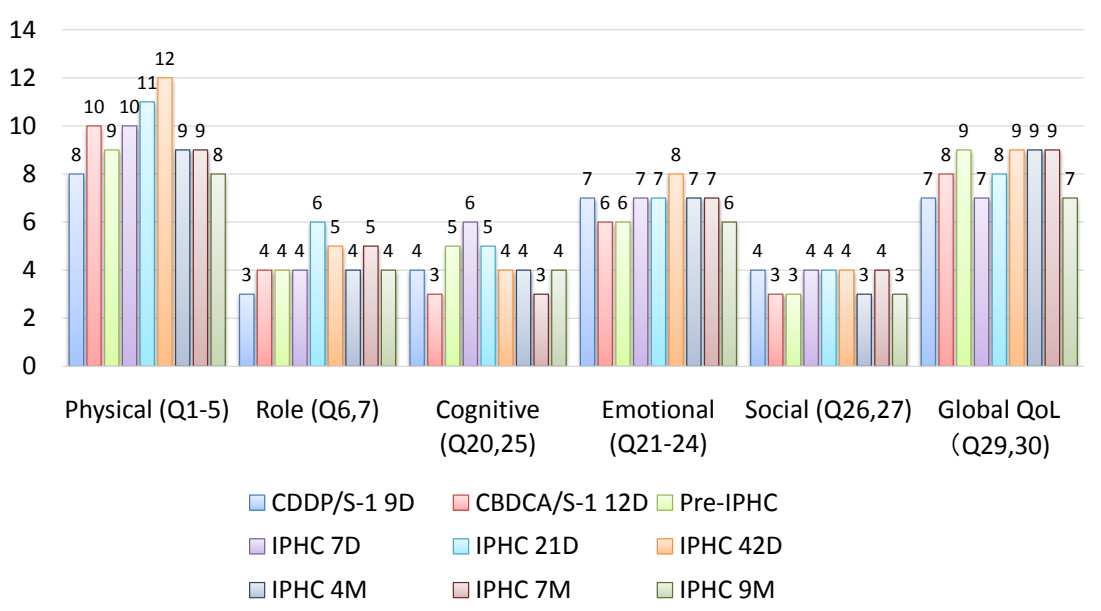

Figure 6. EORTC QLQ-C30 (Functional Scales, Global QoL).

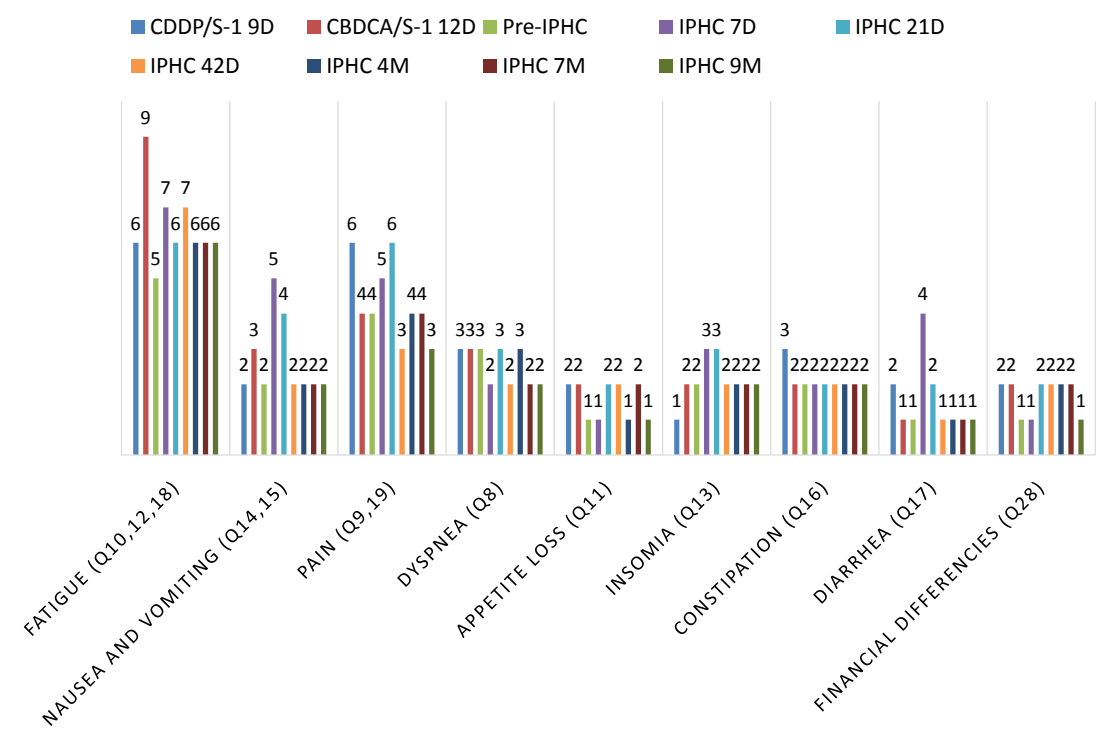

Figure 7. EORTC QLQ-C30 (Symptom scales and single items).

vomiting, pain, and diarrhea after the IPHC were greater than those of the other chemotherapies (CBDCA+S-1 and $\mathrm{CDDP}+\mathrm{S}-1$ ). The degrees of dyspnea, appetite loss, insomnia, constipation, and financial differences, showed similar scale values.

Figure 8 shows the QLQ-LC13 lung module, which addresses specific issues in lung cancer patients, the symptom of dyspnea upon climbing and pain in the chest showed the worse score values compared to the other symptom scales. The symptoms of cough, hemoptysis, rested dyspnea, dyspnea upon walking, dyspnea upon climbing stairs, sore mouth, swallowing, neuropathy, hair loss, pain in the chest, pain in the arm or shoulder, pain elsewhere, and use of analgesics, showed similar score values.

\subsubsection{Outcomes of QOL-ACD}

Figure 9 shows the assessment results of factors of daily-life activities, physical condition, psychology condition, social attitude, and overall QOL. These factor analyses showed similar results among the chemotherapies and IPHC treatments.

Based on the changes in the overall QOL, after the IPHC, it had improved compared to that before the IPHC.

\subsubsection{Outcomes of CDS for Assessing Dyspnea}

Figure 10 shows the results of the changes in the CDS during the IPHC. The CDS is composed of three factors 


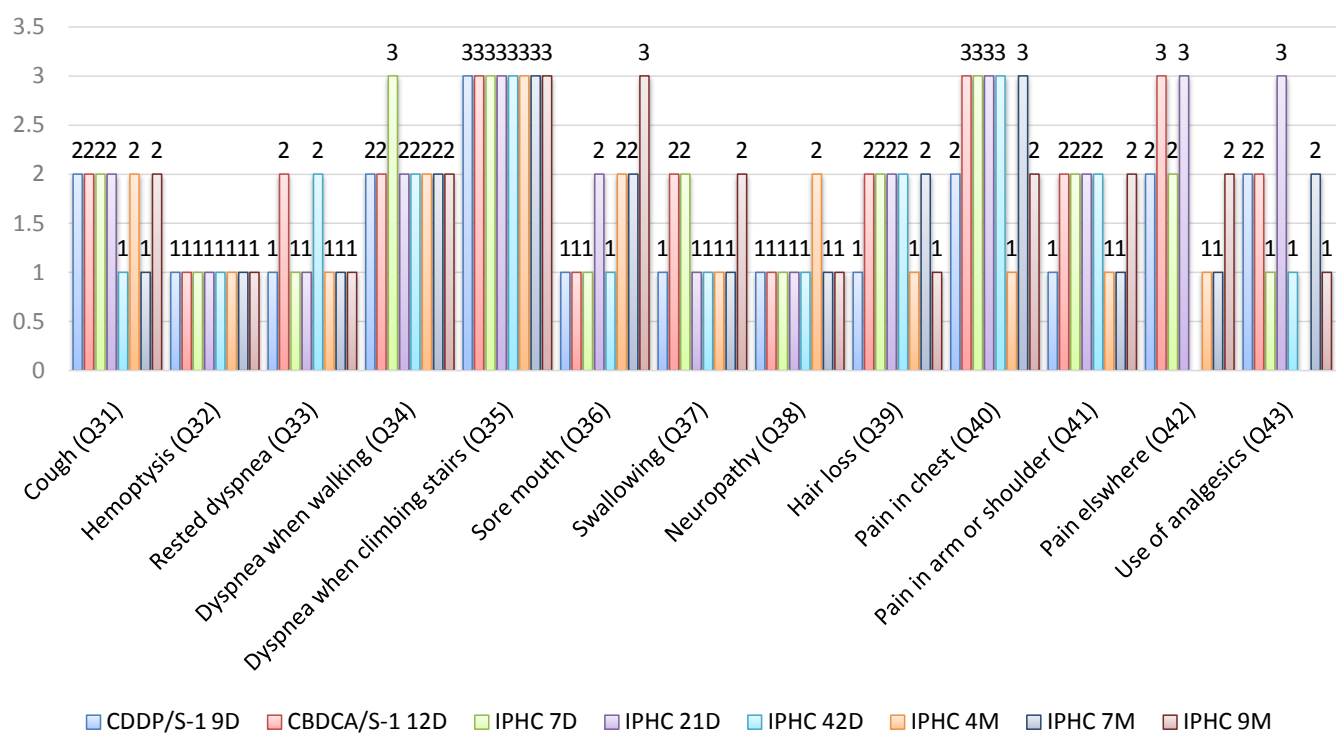

Figure 8. EORTC QLQ-LC13.

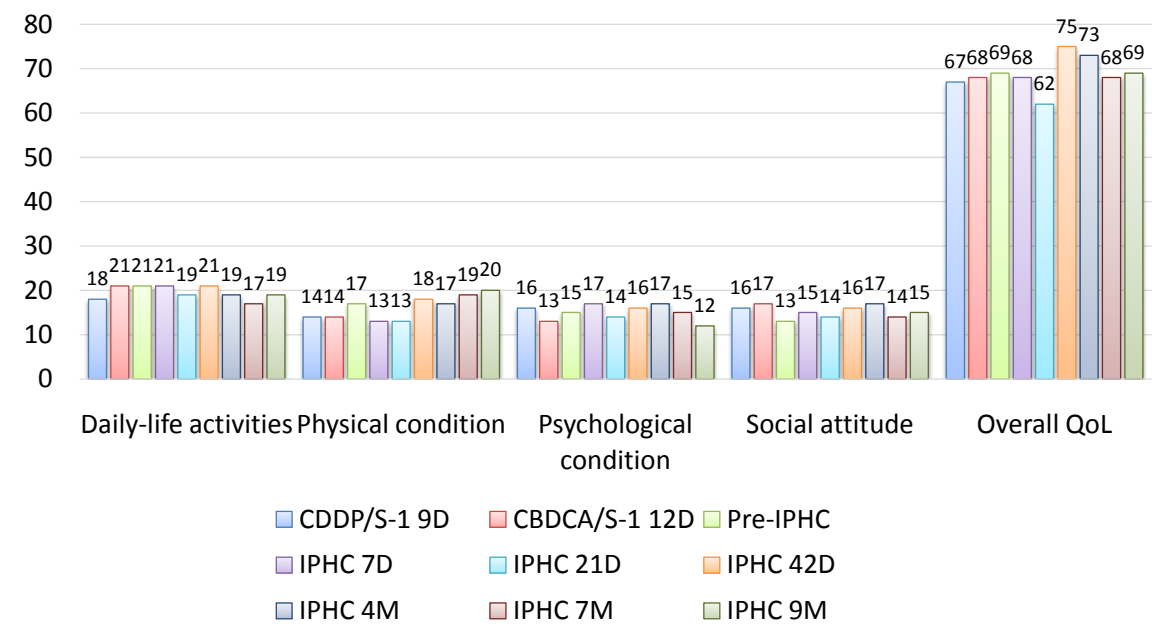

Figure 9. The QOL questionnaire for cancer patients treated with anticancer drugs (QOL-ACD).

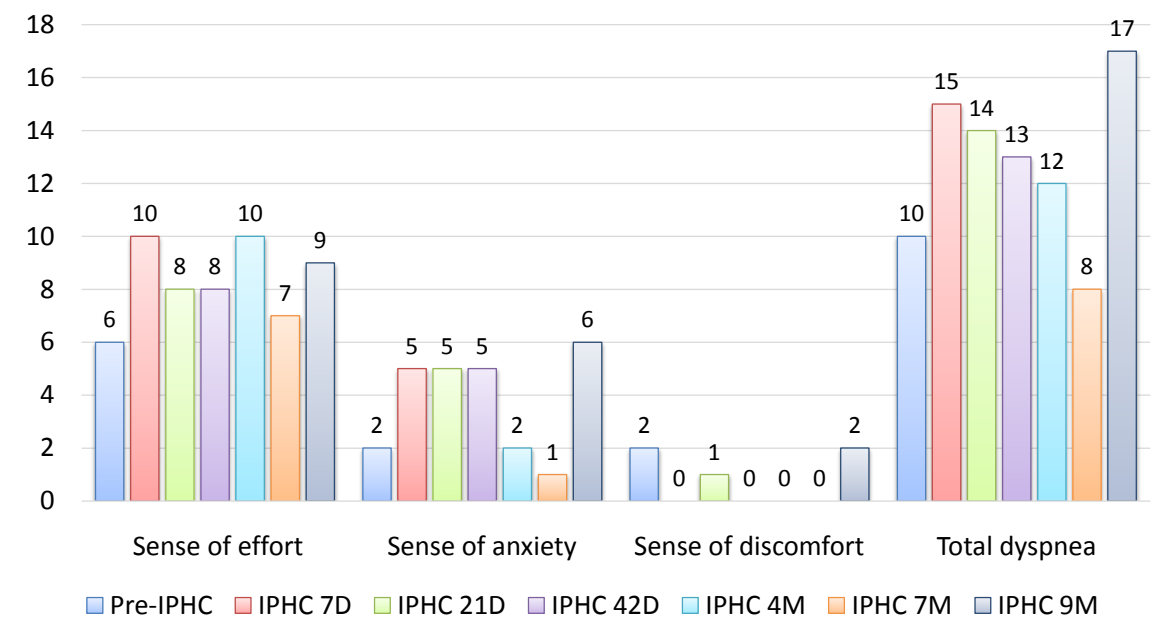

Figure 10. Cancer dyspnea scale (CDS). 
(sense of effort, sense of anxiety, and sense of discomfort) using a factor analysis. The sense of effort was the highest of the three factors, and the sense of discomfort was the lowest. Regarding the total dyspnea, compared to the status of dyspnea before the IPHC, which increased during the period of the IPHC treatment, however, as time passed, the degree of total dyspnea showed a decrease.

\subsubsection{Outcomes of SGRQ}

Figure 11 shows the changes in the SGRQ score during the IPHC treatment, which contained symptoms, activity, impacts, and total scores. For the symptoms score, it displayed an improvement in the symptoms throughout the IPHC treatment period. On day 21 of the IPHC, the activity, impact, and total scores had increased the most, however, gradually improved as time passed.

\subsection{Treatment Effectiveness and Control of Pleural Effusion}

Figure 12(a)-(d) shows the degree of pleural effusion and the change in the reduction for the same slice level of the chest CT scanning. The chest CT scan showed a reduction in the pleural effusion after the chemotherapies and IPHC treatment. The effectiveness of the control of the pleural effusion had been confirmed by the IPHC treatment, the effect of which has been maintained for a year (until December, 2015).

\section{Discussion}

The recent intensive chemotherapy for advanced non-small cell carcinoma has been performed with a platinum doublet regimen, an effective treatment which showed an extension of survival confirmed by the results of some meta-analyses [21] [22]. In the 1990s, new $3^{\text {rd }}$-generation anti-cancer drugs were developed, which included navelbine, paclitaxel, docetaxel, gemcitabine, and pemetrexed. These key new drugs were clinically used with combination with cisplatin or carboplatin. The effectiveness of these platinum doublet therapies has been reported, and the combination therapy has become standard treatment regimens for advanced non-small cell lung cancer [23] [24].

The reason why the regimen of CDDP plus S-1 was selected for our new next-generation IPHC treatment was based on the good results regarding its effectiveness and its feasibility [25]. The clinical trial of the phase II study of S-1 plus cisplatin for non-small cell lung cancer (IIIB/IV) [25] resulted in a high effectiveness for the non-squamous cell lung carcinoma $(n=56)$, a high response rate $(47 \%)(95 \%$ confidence interval $(\mathrm{CI}), 34 \%$ $61 \%$ ), overall survival (11 months), and 1-year survival rate (45\%). The adverse events showed grade 3 and 4 hematologic toxicities, which were neutropenia (29\%) and anemia (22\%), but there were no grade 4 non-hematological toxicities [25]. As the first-line chemotherapy for advanced non-small cell lung cancer (IIIB/IV-staged), the clinical randomized trial of the phase-III study of CDDP+S-1 vs. CDDP + DTX (TCOG 0701, Cisplatin and TS-1 Trial (CATS)) [26], which resulted in an overall survival of 16.1 months in the group of CDDP+S-1 and 17.1 months in the group of CDDP + DTX (hazard ratio (HR), 96.4\%; CI = $1.013(0.837-1.227)$ ). The regimen

SGRQ score

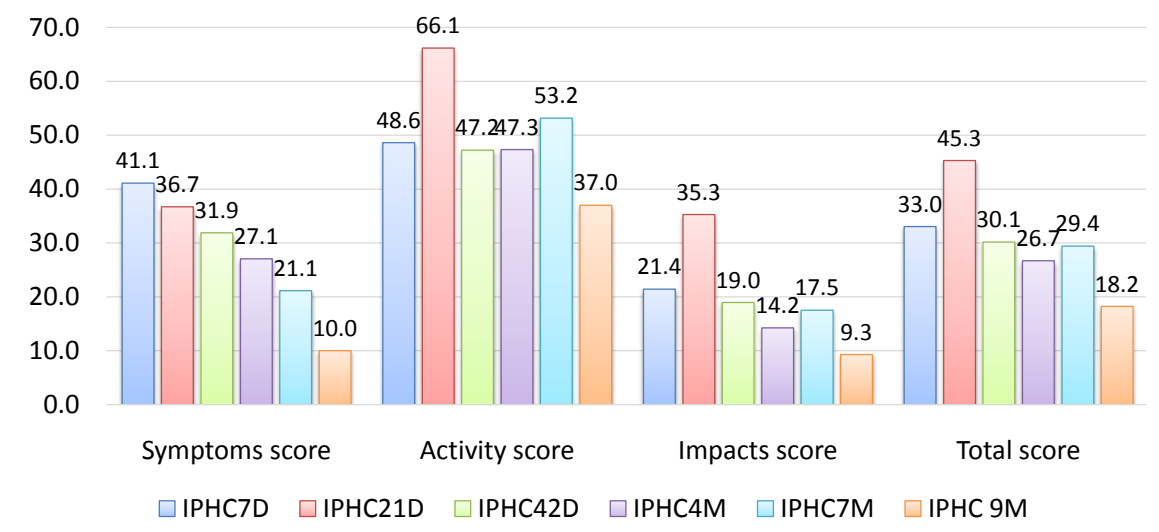

Figure 11. St. george’s respiratory questionnaire total score (SGRQ score). 


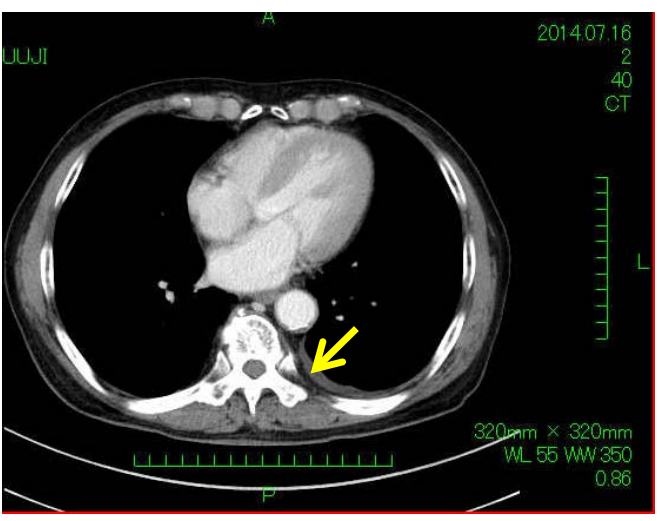

(a)

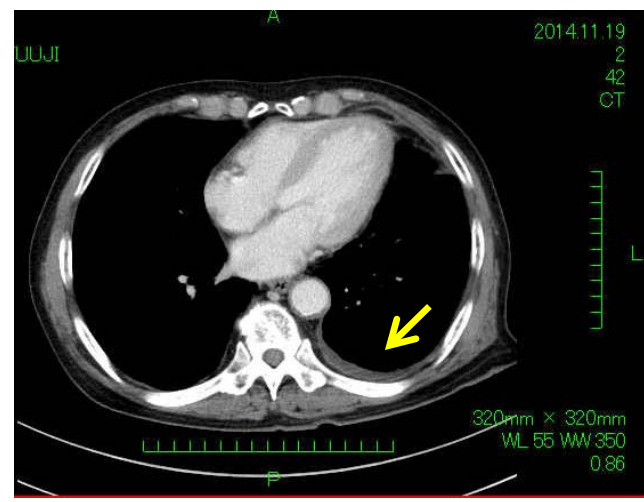

(c)

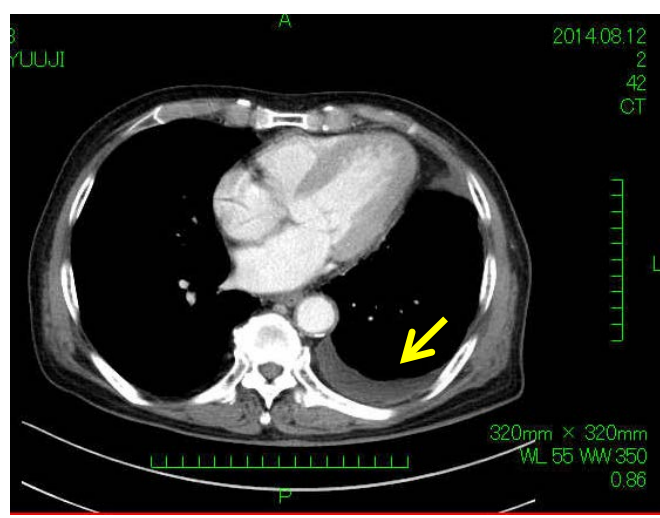

(b)

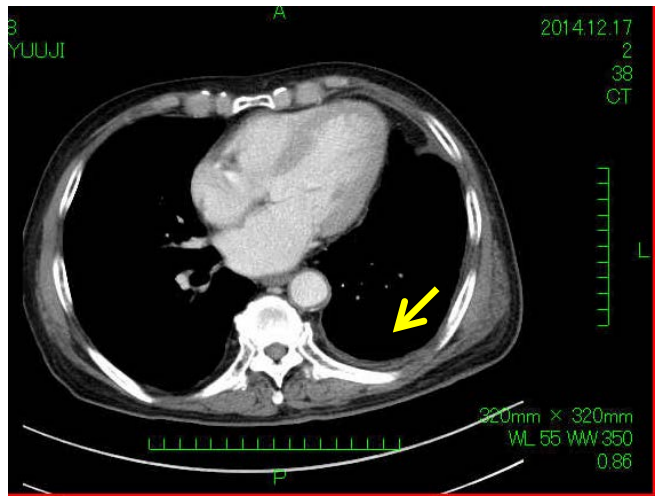

(d)

Figure 12. Transition of the left pleural effusion by the same slice level of the chest CT scanning. The left pleural effusion was reduced and controlled after chemotherapy and IPHC treatment. The effectiveness of IPHC treatment was maintained until December, 2015. (a) Before video-assisted thoracic surgery (in July, 2014). (b) Before systemic chemotherapy (in August, 2014). (c) After two regimens of systemic chemotherapy (in November, 2014). (d) After IPHC (in December, 2014).

of CDDP+S-1 treatment showed a non-inferiority compared to that of the CDDP + DTX treatment, and there was no difference in the progression-free survival (PFS) and response rate (RR) between the two groups. Compared to the regimen of CDDP + DTX, the treatment of CDDP+S-1 resulted in a good feasibility, a low frequency of febrile neutropenia, and good QOL. Based on these results, we adopted the combination of CDDP+ S-1 chemotherapy as a regimen for our new next-generation IPHC.

Systemic chemotherapy is an intravenously dripping method of anti-cancer drugs and/or combination of intravenous infusion and oral medication, however, there is some difference in the approach of the IPHC treatment. As general chemotherapy, an intravenous drip infusion of anti-cancer drugs can systemically reach peripheral sites in the entire body, however, the effect on an intrapleural space was too low compared to that of the IPHC. On the other hand, for the IPHC treatment, the treatment concept is an approach different to the disseminated intrapleural space, where the direct infusion of anti-cancer drugs by the hyperthermic perfusion occures, and that is a good infiltration effect on the inside of the pleural disseminated cells. The disseminated cancer cells and anticancer drugs are in direct contact, and the anti-cancer cytotoxicity is enhanced with hyperthermic effects. The lower the number of disseminated tumor cells, the higher the effectiveness of the hyperthermic chemotherapy.

Regarding the anti-cancer effectiveness of hyperthermia, generally, 1) tumor cells have a high hyperthermic sensitivity compared to those of normal cells; 2) tumor tissue has a high hyperthermic sensitivity selectively depending on the specificity of the surrounding tissue-environment (low $\mathrm{pH}$, hypoxic, fragility of tumor angiogenesis); 3) high hyperthermic sensitivity in the latter phase of the synthesis in the cell cycle; 4) enhancing of the sensitivity of the anti-cancer drug for cancer cells, as denoted, had been performed as a combination therapy with radiation and chemotherapy. The next-generation IPHC treatment has the hybrid effect of the direct cispla- 
tin contact to disseminated lesions and the continuous everyday oral medication of S-1 during the perioperative period.

Which is a better timing for prior to the foregoing of the chemotherapy or IPHC treatment? In this case, two regimens of systemic chemotherapy preceded the IPHC treatment. It took some time to approve our clinical trial by our hospital ethic committee. We obtained the patient's informed consent, and the approved new next-generation IPHC was then performed. Usually, if both therapies could be scheduled, we believe that the IPHC treatment before the systemic chemotherapy would become a better procedure, because the IPHC treatment has some operative risks with invasive surgery and with therapeutic adverse events compared to systemic chemotherapy. As the next-generation IPHC treatment has an aggressive palliative therapeutic concept, the therapy should be performed as soon as possible during the patient's good performance status. If the patient's performance status became worse after the systemic chemotherapy, the IPHC treatment could not be performed because of its surgically invasive high risks. The lower the amount of pleural effusion and the smaller number of disseminated tumor cells, the greater the IPHC treatment would give the patient a better benefit compared to the effectiveness of systemic chemotherapy. Based on these reasons, the IPHC should be performed before the systemic chemotherapy during a patient's good performance status as soon as possible.

For the next candidate modality after the IPHC treatment for this patient, we are intending to follow the patient's good performance status for a while, because the status of good control of the pleural effusion and the patient's good QOL should be maintained for a while. During the observation of the level of tumor markers, the amount of pleural effusion, the local recurrence, distant metastasis, and change in symptoms, a close follow-up should be continued until those failures. If the patient's performance status worsens, the next therapeutic modality should be scheduled. For patients with advanced stage-IV non-small lung cancer, pathologically, they were initially divided into two groups of the squamous cell type or non-squamous cell type. Depending on the results of the types of epidermal growth factor receptor gene mutation and ALK gene translocation, tyrosine kinase inhibitor such as gefitinib, erlotinib, or crizotinib should be scheduled if there were positive findings. On the other hand, for negative findings, another cytotoxic chemotherapy should be selected based on the patient's performance status, age, feasible adverse events, for example, platinum doublet chemotherapy, platinum doublet chemotherapy \pm bevasizumab, non-platinum doublet, monotherapy, oral S-1 medication, etc.

Our traditional, conventional IPHC treatment has not been confirmed by an appropriate clinical trial based on the evaluation items such as tumor response rate and overall survival. For developing our new-generation IPHC, we planned the phase II study, which was newly constructed as a hybrid of our conventional cisplatin-based IPHC and oral continuous S-1 medication. Generally, the aim of the conventional IPHC had a synergistic effectiveness of both an anti-tumor effect of cisplatin and hyperthermic damage to the pleural malignant tumor cells. In the next-generation IPHC, in the addition to the above effectiveness, perioperative continuous oral S-1 medication should enhance the effectiveness of the anti-tumor effect with a low adverse toxicity. The targeted cases of this new next-generation IPHC were thoracic malignances such as unresectable advanced lung cancer with dissemination and carcinomatous pleuritis, intrathoracic recurrencing cases of lung cancer surgery, malignant mesothelioma, sarcoma tumors, metastatic intrapleural tumors due to breast and colon cancers, and other cancers.

\section{Conclusions}

The first clinical trial case of the next-generation IPHC showed Grade 3 adverse events. However, it was an acceptable feasibility compared to the usual platinum doublet chemotherapy.

The pleural effusion had been reduced and the effectiveness of the controlied pleural effusion had been maintained, and the patient's QOL had been preserved.

\section{Acknowledgements}

This study was supported by a Grant-in-Aid for Clinical Research from the Miyazaki University Hospital.

\section{References}

[1] Jemal, A., Bray, F., Center, M.M., Ferlay, J., Ward, E. and Forman, D. (2011) Global Cancer Statistics. CA: Cancer Journal for Clinicians, 61, 69-90. http://dx.doi.org/10.3322/caac.20107 
[2] Masuda, M., Kuwano, H., Okumura, M., Jun Amano, J., Arai, H., Endo, S., Doki, Y., Kobayashi, K., Motomura, N., Nishida, N., Saiki, Y., Tanaka, F., Tanemoto, K., Toh, Y. and Yokomise, H., Committee for Scientific Affairs, The Japanese Association for Thoracic Surgery (2014) Thoracic and Cardiovascular Surgery in Japan during 2012. Annual Report by the Japanese Association for Thoracic Surgery. General Thoracic and Cardiovascular Surgery, 62, 734-764. http://dx.doi.org/10.1007/s11748-014-0464-0

[3] Goldstraw, P., Crowley, J., Chansky, K., Giroux, D.J., Groome, P.A., Rami-Porta, R., Postmus, P.E., Rusch, V. and Sobin, L. (2007) International Association for the Study of Lung Cancer International Staging Committee, Participating Institutions. The IASLC Lung Cancer Staging Project: Proposals for the Revision of the TNM Stage Groupings in the Forthcoming (Seventh) Edition of the TNM Classification of Malignant Tumours. Journal of Thoracic Oncology, 2, 706-714. http://dx.doi.org/10.1097/JTO.0b013e31812f3c1a

[4] Matsuzaki, Y., Shibata, K., Yoshioka, M., Inoue, M., Sekiya, R., Onitsuka, T., Iwamoto, I. and Koga, Y. (1995) Intrapleural Perfusion Hyperthermo-Chemotherapy for Malignant Pleural Dissemination and Effusion. The Annals of Thoracic Surgery, 59, 127-131. http://dx.doi.org/10.1016/0003-4975(94)00614-D

[5] Matsuzaki, Y., Edagawa, M., Shimizu, T., Hara, M., Tomita, M., Ayabe, T. and Onitsuka, T. (2004) Intrapleural Hyperthermic Perfusion with Chemotherapy Increases Apoptosis in Malignant Pleuritis. The Annals of Thoracic Surgery, 78, 1769-1772. http://dx.doi.org/10.1016/j.athoracsur.2004.05.025

[6] Matsuzaki, Y., Tomita, M., Shimizu, T., Hara, M., Ayabe, T. and Onitsuka, T. (2008) Induction of Apoptosis by Intrapleural Perfusion Hyperthermo-Chemotherapy for Malignant Pleural Mesothelioma. The Annals of Thoracic Surgery, 14, 161-165.

[7] Ayabe, T., Tomita, M., Chosa, E., Kawagoe, K. and Nakamura, K. (2015) Pneumonectomy of Primary Pulmonary Angiosarcoma with Malignant Effusion and Intrapleural Hypotonic Hyperthermic Chemotherapy: Case Report and Review of the Literature. Journal of Cancer Therapy, 6, 227-236. http://dx.doi.org/10.4236/jct.2015.62025

[8] Trotti, R., Carratelli, M. and Barbieri, M. (2002) Performance and Clinical Application of a New, Fast Method for the Detection of Hydroperoxides in Serum. Panminerva Medica, 44, 37-40.

[9] Alberti, A., Bolognini, L., Macciantelli, D. and Carratelli, M. (2000) The Radical Cation of N,N-Diethyl-para-phenylendiamine: A Possibleindicator of Oxidative Stress in Biological Samples. Research on Chemical Intermediates, 26, 253-267.

[10] Cornelli, U., Terranova, R., Luca, S., Cornelli, M. and Alberti, A. (2001) Bioavailability and Antioxidant Activity of Some Food Supplements in Men and Women Using the D-Roms Test as a Marker of Oxidative Stress. Journal of Nutrition, 131, 3208-3211.

[11] Tsuchiya, M., Sato, E., Inoue, M. and Asada, A. (2008) Open Abdominal Surgery Increases Intraoperative Oxidative Stress: Can It Be Prevented? Anesthesia \& Analgesia, 107, 1946-1952. http://dx.doi.org/10.1213/ane.0b013e318187c96b

[12] Earle, C.C. (2004) Outcomes Research in Lung Cancer. Journal of the National Cancer Institute Monographs, 33, 56-77. http://dx.doi.org/10.1093/jncimonographs/lgh001

[13] Aaronson, N.K., Ahmedzai, S., Bergman, B., Bullinger, M., Cull, A., Duez, N.J., Filiberti, A., Flechtner, H., Fleishman, S.B. and de Haes, J.C. (1993) The European Organization for Research and Treatment of Cancer QLQ-C30: A Quality-of-Life Instrument for Use in International Clinical Trials in Oncology. Journal of the National Cancer Institute, 85, 365-376. http://dx.doi.org/10.1093/jnci/85.5.365

[14] Kurihara, M., Shimizu, H., Tsuboi, K., Kobayashi, K., Murakami, M., Eguchi, K. and Shimozuma, K. (1999) Development of Quality of Life Questionnaire in Japan: Quality of Life Assessment of Cancer Patients Receiving Chemotherapy. Psycho-Oncology, 8, 355-363. http://dx.doi.org/10.1002/(SICI)1099-1611(199907/08)8:4<355::AID-PON401>3.0.CO;2-I

[15] Matsumoto, T., Ohashi, Y., Morita, S., Kobayashi, K., Shibuya, M., Yamaji, Y., Eguchi, K., Fukuoka, M., Nagao, K., Nishiwaki, Y. and Niitani, H. (2002) CPT-11 Lung Cancer Study Groups West and East. The Quality of Life Questionnaire for Cancer Patients Treated with Anticancer Drugs (QOL-ACD): Validity and Reliability in Japanese Patients with Advanced Non-Small-Cell Lung Cancer. Quality of Life Research, 11, 483-493. http://dx.doi.org/10.1023/A:1015614505929

[16] Bonomi, A.E., Cella, D.F., Hahn, E.A., Bjordal, K., Sperner-Unterweger, B., Gangeri, L., Bergman, B., Willems-Groot, J., Hanquet, P. and Zittoun, R. (1996) Multilingual Translation of the Functional Assessment of Cancer Therapy (FACT) Quality of Life Measurement System. Quality of Life Research, 5, 309-320. http://dx.doi.org/10.1007/BF00433915

[17] Maneesriwongul, W. and Dixon, J.K. (2004) Instrument Translation Process: A Methods Review. Journal of Advanced Nursing, 48, 175-186. http://dx.doi.org/10.1111/j.1365-2648.2004.03185.X

[18] White, M. and Elander, G. (1992) Translation of an Instrument. The US-Nordic Family Dynamics Nursing Research Project. Scandinavian Journal of Caring Sciences, 6, 161-164. http://dx.doi.org/10.1111/j.1471-6712.1992.tb00145.X 
[19] Tanaka, K., Akechi, T., Okuyama, T., Nishiwaki, Y. and Uchitomi, Y. (2000) Development and Validation of the Cancer Dyspnoea Scale: A Multidimensional, Brief, Self-Rating Scale. British Journal of Cancer, 82, 800-805. http://dx.doi.org/10.1054/bjoc.1999.1002

[20] Jones, P.W., Quirk, F.H., Baveystock, C.M. and Littlejohns, P. (1992) A Self-Complete Measure of Health Status for Chronic Airflow Limitation. The American Review of Respiratory Disease, 145, 1321-1327. http://dx.doi.org/10.1164/ajrccm/145.6.1321

[21] Non-Small Cell Lung Cancer Collaborative Group (1995) Chemotherapy in Non-Small Cell Lung Cancer: A Meta-Analysis Using Updated Data on Individual Patients from 52 Randomised Clinical Trials. BMJ, 311, 899-909. http://www.ncbi.nlm.nih.gov/pmc/articles/PMC2550915/pdf/bmj00613-0017.pdf http://dx.doi.org/10.1136/bmj.311.7010.899

[22] Marino, P., Pampallona, S., Preatoni, A., Cantoni, A. and Invernizzi, F. (1994) Chemotherapy vs Supportive Care in Advanced Non-Small-Cell Lung Cancer. Results of a Meta-Analysis of the Literature. Chest, 106, 861-865. http://dx.doi.org/10.1378/chest.106.3.861

[23] Wozniak, A.J., Crowley, J.J., Balcerzak, S.P., Weiss, G.R., Spiridonidis, C.H., Baker, L.H., Albain, K.S., Kelly, K., Taylor, S.A., Gandara, D.R. and Livingston, R.B. (1998) Randomized Trial Comparing Cisplatin with Cisplatin plus Vinorelbine in the Treatment of Advanced Non-Small-Cell Lung Cancer: A Southwest Oncology Group Study. Journal of Clinical Oncology, 16, 2459-2465.

[24] Yana, T., et al. (2002) New Chemotherapy Agent plus Platinum for Advanced Non-Small Cell Lung Cancer: A Meta-Analysis. Proceedings of American Society of Clinical Oncology, 21, 328a.

[25] Ichinose, Y., Yoshimori, K., Sakai, H., Nakai, Y., Sugiura, T., Kawahara, M. and Niitani, H. (2004) S-1 plus Cisplatin Combination Chemotherapy in Patients with Advanced Non-Small Cell Lung Cancer: A Multi-Institutional Phase II Trial. Clinical Cancer Research, 10, 7860-7864. http://dx.doi.org/10.1158/1078-0432.CCR-04-1200

[26] Kubota, K., Sakai, H., Katakami, N., Nishio, M., Inoue, A., Okamoto, H., Isobe, H., Kunitoh, H., Takiguchi, Y., Kobayashi, K., Nakamura, Y., Ohmatsu, H., Sugawara, S., Minato, K., Fukuda, M., Yokoyama, A., Takeuchi, M., Michimae, H., Gemma, A. and Kudoh, S., Tokyo Cooperative Oncology Group (2015) A Randomized Phase III Trial of Oral S-1 plus Cisplatin versus Docetaxel plus Cisplatin in Japanese Patients with Advanced Non-Small-Cell Lung Cancer: TCOG0701 CATS Trial. Annals of Oncology, 26, 1401-1408. http://dx.doi.org/10.1093/annonc/mdv190

\author{
Abbreviations \\ IPHC: Intrapleural Hyperthermic Chemotherapy \\ QOL: Quality of Life \\ TNM: Tumor, Lymph nodes, Metastasis \\ CDDP: Cispatin \\ CBDCA: Carboplatin \\ DTX: Docetaxel
}

